\title{
Ground states of nonlinear Schrödinger equations with potentials
}

\section{Solutions d'énergie minimale des équations de Schrödinger non-linéaires avec potentiel}

\author{
Yongqing Li $^{\text {a, } 1}$, Zhi-Qiang Wang a,b,*, Jing Zeng ${ }^{\text {a }}$ \\ a School of Mathematics and Computer Sciences, Fujian Normal University, Fuzhou, 350007, PR China \\ ${ }^{\mathrm{b}}$ Department of Mathematics and Statistics, Utah State University, Logan, UT 84322, USA
}

Received 1 September 2005; accepted 31 January 2006

Available online 7 July 2006

\section{Abstract}

In this paper we study the nonlinear Schrödinger equation:

$$
\left\{\begin{array}{l}
-\Delta u+V(x) u=f(x, u) \\
u \in H^{1}\left(\mathbb{R}^{N}\right)
\end{array}\right.
$$

We give general conditions which assure the existence of ground state solutions. Under a Nehari type condition, we show that the standard Ambrosetti-Rabinowitz super-linear condition can be replaced by a more natural super-quadratic condition.

๑ 2006 L'Association Publications de l'Institut Henri Poincaré. Published by Elsevier B.V. All rights reserved

\section{Résumé}

Dans cet article nous étudions l'équation non-linéaire de Schrödinger :

$$
\left\{\begin{array}{l}
-\Delta u+V(x) u=f(x, u) \\
u \in H^{1}\left(\mathbb{R}^{N}\right)
\end{array}\right.
$$

Nous donnons les conditions générales qui garantissent l'existence de solutions d'énergie minimale. Sous une condition de type Nehari, nous démontrons que la condition super-linéaire d'Ambrosetti-Rabinowitz peut être remplacée par une condition superquadratique plus naturelle.

๔ 2006 L'Association Publications de l'Institut Henri Poincaré. Published by Elsevier B.V. All rights reserved

MSC: 35B05; 35J60

Keywords: Nonlinear Schrödinger equations; Ground state solutions; The Ambrosetti-Rabinowitz condition

\footnotetext{
* Corresponding author.

E-mail addresses: zhi-qiang.wang@math.usu.edu, wang@math.usu.edu (Z.-Q. Wang).

1 Supported in part by NNSF of China (10161010), Fujian Provincial Natural Science Foundation of China (A0410015), and the Ky and Yu-Fen Fan Fund from AMS.
} 


\section{Introduction}

We study the nonlinear Schrödinger equation with potentials:

$$
\left\{\begin{array}{l}
-\Delta u+V(x) u=f(x, u) \\
u \in H^{1}\left(\mathbb{R}^{N}\right)
\end{array}\right.
$$

We are concerned with the existence of ground state solutions, i.e., solutions corresponding to the least positive critical value of the variational functional:

$$
\Phi(u)=\frac{1}{2} \int_{\mathbb{R}^{N}}\left(|\nabla u|^{2}+V(x) u^{2}\right) \mathrm{d} x-\int_{\mathbb{R}^{N}} F(x, u) \mathrm{d} x,
$$

where $F(x, u)=\int_{0}^{u} f(x, t) \mathrm{d} t$.

To establish the existence of ground states, usually besides the growth condition on the nonlinearity and a Nehari type condition, the following superlinear condition due to Ambrosetti-Rabinowitz (e.g., [2,12]) is assumed:

(AR) There is $\mu>2$ such that for $u \neq 0$ and $x \in \mathbb{R}^{N}$,

$$
0<\mu F(x, u) \leqslant u f(x, u),
$$

where $F(x, u)=\int_{0}^{u} f(x, t) \mathrm{d} t$.

This condition implies that for some $C>0, F(x, u) \geqslant C|u|^{\mu}$.

In this paper we show that a weaker and more natural version suffices to assure the existence of a ground state solution. Instead of (AR) we assume the following super-quadratic condition

(SQ) $\lim _{|u| \rightarrow \infty} \frac{F(x, u)}{u^{2}}=\infty$, uniformly in $x$.

We always assume $V(x) \in C\left(\mathbb{R}^{N}, \mathbb{R}\right), \inf _{\mathbb{R}^{N}} V(x)>0$. We consider two cases of the potentials, one is periodic, i.e., the $x$-dependence is periodic; the other is when $V$ has a bounded potential well in the sense that $\lim _{|x| \rightarrow \infty} V(x)$ exists and is equal to $\sup _{\mathbb{R}^{N}} V$. The results will be stated and proved in Sections 2 and 3.

We would like to mention earlier results on existence of entire solutions of Schrödinger type equations with or without potentials which was studied in $[3,4,9,10]$ (see references therein). In recent years there have been intensive studies on semiclassical states for nonlinear Schrödinger equations for which in Eq. (1.1) there is a small parameter corresponding to the Plank constant. We refer [1] for references in this direction. Our results do not require smallness of such a parameter. A recent result in [5] is in similar spirit of our Theorem 3.1; but the conditions in [5] and ours are mutually non-inclusive and the methods are different.

For (1.1) in bounded domains or if the potential function $V(x)$ possesses certain compactness condition, one can prove (1.1) have certain solutions. In [8] Liu and Wang first used (SQ) to get the bounds of minimizing sequences on the Nehari manifold, and under coercive condition of $V(x)$ they proved the existence of three solutions: one positive, one negative, and one sign-changing. The results in this current paper are natural generalizations of that in [8] to noncompact cases. In the two cases we do not have compact embedding, which is the main difficulty in this paper. We shall make use of a combination of the techniques in $[8,7]$ with applications of the concentration-compactness principle of Lions $[6,11,12]$.

\section{The periodic case}

We consider weak solutions of

$$
\left\{\begin{array}{l}
-\Delta u+V(x) u=f(x, u), \\
u \in H^{1}\left(\mathbb{R}^{N}\right) .
\end{array}\right.
$$

We need the following assumptions:

$\left(V_{1}\right) V(x) \in C\left(\mathbb{R}^{N}, \mathbb{R}\right), \inf _{\mathbb{R}^{N}} V(x) \geqslant V_{0}>0 . V(x)$ is 1-periodic in each of $x_{1}, x_{2}, \ldots, x_{N}$. 
( $\left.f_{1}\right) f(x, t) \in C^{1}$ is 1-periodic in each of $x_{1}, x_{2}, \ldots, x_{N}, f_{t}$ is a Caratheodory function and there exists $C>0$, such that

$$
\left|f_{t}(x, t)\right| \leqslant C\left(1+|t|^{2^{*}-2}\right), \quad \lim _{|t| \rightarrow \infty} \frac{|f(x, t)|}{|t|^{2^{*}-1}}=0, \quad \text { uniformly in } x \in \mathbb{R}^{N} .
$$

$\left(f_{2}\right) \quad f(x, t)=\mathrm{o}(|t|)$, as $|t| \rightarrow 0$, uniformly in $x$.

(f $\left.f_{3}\right) \lim _{|t| \rightarrow \infty} \frac{F(x, t)}{t^{2}}=\infty$, uniformly in $x$.

$\left(f_{4}\right) \frac{f(x, t)}{|t|}$ is strictly increasing in $t$.

Here $2^{*}=\frac{2 N}{N-2}$ for $N \geqslant 3$. For $N=1,2$ we assume there is $q>2$ in the place of $2^{*}$ in $\left(f_{1}\right)$. We work in Hilbert space $X=\left\{u \in H^{1}\left(\mathbb{R}^{N}\right) ; \int_{\mathbb{R}^{N}} V(x) u^{2} \mathrm{~d} x<\infty\right\}$, with norm $\|u\|^{2}=\int_{\mathbb{R}^{N}}\left(|\nabla u|^{2}+V(x) u^{2}\right) \mathrm{d} x$. The functional associated with Eq. (1.1) is

$$
\Phi(u)=\frac{1}{2} \int_{\mathbb{R}^{N}}\left(|\nabla u|^{2}+V(x) u^{2}\right) \mathrm{d} x-\int_{\mathbb{R}^{N}} F(x, u) \mathrm{d} x, \quad u \in X .
$$

Define

$$
\gamma(u)=\int_{\mathbb{R}^{N}}\left(|\nabla u|^{2}+V(x) u^{2}\right) \mathrm{d} x-\int_{\mathbb{R}^{N}} f(x, u) u \mathrm{~d} x .
$$

Theorem 2.1. Under assumptions $\left(V_{1}\right),\left(f_{1}\right)-\left(f_{4}\right)$ Eq. (1.1) has a weak solution $u \in X$, such that $\Phi(u)=c>0, c$ is defined as

$$
c=\inf _{\mathcal{N}} \Phi(u)
$$

where $\mathcal{N}=\{u \in X: u \neq 0, \gamma(u)=0\}$.

First we need a few lemmas.

Lemma 2.2. Let $\left(u_{n}\right)$ be a minimizing sequence for $c$. Then

(i) There is $\beta>0$ such that $\liminf _{n \rightarrow \infty}\left\|u_{n}\right\| \geqslant \beta$.

(ii) $\left(u_{n}\right)$ is bounded in $X$.

(iii) For a subsequence, up to translations, $u_{n}$ converges weakly to $u \neq 0$.

Proof. (i) The proof is similar to the case with (AR) satisfied. We omit its proofs (see [12]).

(ii) Let $\left(u_{n}\right)$ be a minimizing sequence of $c$. If $\left(u_{n}\right)$ is not bounded, we define $v_{n}=u_{n} /\left\|u_{n}\right\|$, so $\left\|v_{n}\right\|=1$. Passing to a subsequence, we may assume, $v_{n} \rightarrow v$ in $X, v_{n} \rightarrow v$ in $L_{\text {loc }}^{p}\left(\mathbb{R}^{N}\right), 2 \leqslant p<2^{*}, v_{n} \rightarrow v$ a.e. on $\mathbb{R}^{N}$.

If $v \neq 0$, we have

$$
\frac{1}{2}-\int_{\mathbb{R}^{N}} \frac{F\left(x, u_{n}\right)}{u_{n}^{2}} v_{n}^{2} \mathrm{~d} x=\frac{c+\mathrm{o}(1)}{\left\|u_{n}\right\|^{2}}>0 .
$$

By Fadou's lemma and $\left(f_{3}\right)$ we have a contradiction as follows,

$$
\frac{1}{2} \geqslant \liminf _{n \rightarrow \infty} \int_{\mathbb{R}^{N}} \frac{F\left(x, u_{n}\right)}{u_{n}^{2}} v_{n}^{2} \mathrm{~d} x \geqslant \int_{\mathbb{R}^{N}} \liminf _{n \rightarrow \infty} \frac{F\left(x, u_{n}\right)}{u_{n}^{2}} v_{n}^{2} \mathrm{~d} x=\infty .
$$

If $v=0$, we take $y_{n}=\left(y_{n}^{1}, y_{n}^{2}, \ldots, y_{n}^{N}\right) \in \mathbb{N}^{N}$ with all $y_{n}^{i}(1 \leqslant i \leqslant N)$ being integers. Define translations of $v_{n}$ by $w_{n}(x)=v_{n}\left(x+y_{n}\right)$. Since $V(x)$ and $f(x, u)$ are periodic, we have $\left\|w_{n}\right\|=\left\|v_{n}\right\|=1,\left|w_{n}\right|_{p}=\left|v_{n}\right|_{p}$, and $\Phi\left(w_{n}\right)=$ $\Phi\left(v_{n}\right)$. Passing to a subsequence, we have $w_{n} \rightarrow w$ in $H^{1}\left(\mathbb{R}^{N}\right), w_{n} \rightarrow w$ in $L_{\text {loc }}^{p}\left(\mathbb{R}^{N}\right), 2 \leqslant p<2^{*}, w_{n} \rightarrow w$ a.e. 
on $\mathbb{R}^{N}$. If there exist $y_{n}$, such that $w_{n} \rightarrow w \neq 0$, we will get a contradiction as the case of $v \neq 0$. If for any $y_{n}, w_{n} \rightarrow 0$, we will get a contradiction by proving $v_{n} \rightarrow 0$ in $L^{p}\left(\mathbb{R}^{N}\right)$. In this case, we claim for all $p \in\left(2,2^{*}\right)$,

$$
\limsup _{n \rightarrow \infty} \int_{y \in \mathbb{R}^{N}}\left|v_{n}\right|^{p} \mathrm{~d} x=0 .
$$

If this is not true, there exists $p \in\left(2,2^{*}\right), \delta>0$,

$$
\limsup _{n \rightarrow \infty} \int_{y \in \mathbb{R}^{N}}\left|v_{n}\right|^{p} \mathrm{~d} x \geqslant \delta>0,
$$

then there exists $z_{n} \in \mathbb{R}^{N}$ such that, $\lim _{n \rightarrow \infty} \int_{B_{2}\left(z_{n}\right)}\left|v_{n}\right|^{p} \mathrm{~d} x \geqslant \delta / 2>0$. We can choose $y_{n} \in \mathbb{N}^{N} \in B_{2}\left(z_{n}\right)$ such that $B_{1}\left(y_{n}\right) \subset B_{2}\left(z_{n}\right)$ and

$$
\lim _{n \rightarrow \infty} \int_{B_{1}\left(y_{n}\right)}\left|v_{n}\right|^{p} \mathrm{~d} x \geqslant \frac{\delta}{2}>0,
$$

we have $\lim _{n \rightarrow \infty} \int_{B_{1}(0)}\left|w_{n}\right|^{p} \mathrm{~d} x \geqslant \delta / 2>0$, that is $w_{n} \rightarrow w \neq 0$, a contradiction.

By Lions Lemma (cf. [12, Lemma 1.21]), we get $v_{n} \rightarrow 0$ in $L^{p}\left(\mathbb{R}^{N}\right), p \in\left(2,2^{*}\right)$. Fix $p \in\left(2,2^{*}\right)$. By $\left(f_{1}\right)$ and $\left(f_{2}\right)$, for any $\varepsilon>0$ there is $C_{\varepsilon}>0$ such that $|f(x, u)| \leqslant \varepsilon\left(|u|+|u|^{2^{*}-1}\right)+C_{\varepsilon}|u|^{p-1}$. Then $|F(x, u)| \leqslant \varepsilon\left(|u|^{2}+|u|^{2^{*}}\right)+$ $C_{\varepsilon}|u|^{p}$. Then fixing an $R>\sqrt{2 c}$, using Lebesgue Dominated Convergence theorem, we have

$$
\lim _{n \rightarrow \infty} \int_{\mathbb{R}^{N}} F\left(x, R v_{n}\right) \mathrm{d} x=\int_{\mathbb{R}^{N}} \lim _{n \rightarrow \infty} F\left(x, R v_{n}\right) \mathrm{d} x=0 .
$$

Since by $\left(f_{4}\right), \Phi\left(t u_{n}\right) \leqslant \Phi\left(u_{n}\right)$ for $t \geqslant 0$ we thus have

$$
c+\mathrm{o}(1)=\Phi\left(u_{n}\right) \geqslant \Phi\left(R v_{n}\right)=\frac{1}{2} R^{2}-\int_{\mathbb{R}^{N}} F\left(x, R v_{n}\right) \mathrm{d} x,
$$

which is a contradiction. Thus $\left(u_{n}\right)$ is bounded.

(iii) We can assume $u_{n}$ weakly converges to $u$. To show $u \neq 0$, again we define translations of $u_{n}$ as above, assume $y_{n}=\left(y_{n}^{1}, y_{n}^{2}, \ldots, y_{n}^{N}\right) \in \mathbb{N}^{N}$, with all $y_{n}^{i}(1 \leqslant i \leqslant N)$ being integers. $u_{n}^{y_{n}}=u_{n}\left(x+y_{n}\right)$ are all possible translation of $u_{n}$. If for some $y_{n} \subset \mathbb{N}^{N}, u_{n}^{y_{n}} \rightarrow u \neq 0$ we are done. If for any $y_{n} \subset \mathbb{N}^{N}, u_{n}^{y_{n}} \rightarrow 0$, by similar argument as above we can prove $u_{n} \rightarrow 0$ in $L^{p}\left(\mathbb{R}^{N}\right), p \in\left(2,2^{*}\right)$. Then as $n \rightarrow \infty, \int_{\mathbb{R}^{N}} u_{n} f\left(x, u_{n}\right) \mathrm{d} x \rightarrow 0$. Thus by (i) we have a contradiction:

$$
0<\beta \leqslant\left\|u_{n}\right\|^{2}=\int_{\mathbb{R}^{N}} u_{n} f\left(x, u_{n}\right) \mathrm{d} x \rightarrow 0, \quad \text { as } n \rightarrow \infty .
$$

Lemma 2.3. For each $u \in X \backslash\{0\}$, there exists unique $t=t(u)>0$, such that $t u \in \mathcal{N}$.

This is similar to the case of assuming (AR), we omit it.

Lemma 2.4. Let $\left(u_{n}\right) \subset X$ be a sequence such that $\gamma\left(u_{n}\right) \rightarrow 0$ and $\int_{\mathbb{R}^{N}} f\left(x, u_{n}\right) u_{n} \rightarrow a>0$ as $n \rightarrow \infty$. Then exist $t_{n}>0$ such that $t_{n} u_{n} \in \mathcal{N}, t_{n} \rightarrow 1$, as $n \rightarrow \infty$.

Proof. Since $u_{n} \neq 0$, by Lemma 2.3, there exists only one $t_{n}>0$, such that $t_{n} u_{n} \in \mathcal{N}$, i.e.

$$
t_{n}^{2} \int_{\mathbb{R}^{N}}\left(\left|\nabla u_{n}\right|^{2}+V(x)\left|u_{n}\right|^{2}\right) \mathrm{d} x-\int_{\mathbb{R}^{N}} f\left(x, t_{n} u_{n}\right) t_{n} u_{n} \mathrm{~d} x=0 .
$$

By $\left(f_{1}\right)$ and $\left(f_{2}\right),|f(x, u) u| \leqslant \varepsilon\left(|u|^{2}+|u|^{2^{*}}\right)+C_{\varepsilon}|u|^{p}$, we see $t_{n}$ cannot go zero, that is $t_{n} \geqslant t_{0}>0$. By $\left(f_{4}\right)$, $f(x, u) u \geqslant 2 F(x, u)$. If $t_{n} \rightarrow \infty$, we get

$$
a+\mathrm{o}(1)=\int_{\mathbb{R}^{N}}\left(\left|\nabla u_{n}\right|^{2}+V(x)\left|u_{n}\right|^{2}\right) \mathrm{d} x=\int_{\mathbb{R}^{N}} \frac{f\left(x, t_{n} u_{n}\right) t_{n} u_{n}}{t_{n}^{2}} \mathrm{~d} x \geqslant 2 \int_{\mathbb{R}^{N}} \frac{F\left(x, t_{n} u_{n}\right)}{t_{n}^{2} u_{n}^{2}} u_{n}^{2} \mathrm{~d} x .
$$


By the condition, up to translations, $u_{n} \rightarrow u \neq 0$ a.e. in $\mathbb{R}^{N}$. We have

$$
\int_{\mathbb{R}^{N}} \frac{F\left(x, t_{n} u_{n}\right)}{t_{n}^{2} u_{n}^{2}} u_{n}^{2} \mathrm{~d} x \rightarrow+\infty, \quad \text { as } n \rightarrow \infty
$$

a contradiction. Thus $0<t_{0} \leqslant t_{n} \leqslant C$. Assume $t_{n} \rightarrow T$, now we claim $T=1$. Since $t_{n} u_{n} \in \mathcal{N}$, by $\gamma\left(u_{n}\right) \rightarrow 0$ we have

$$
\int_{\mathbb{R}^{N}}\left(\left|\nabla u_{n}\right|^{2}+V(x)\left|u_{n}\right|^{2}\right) \mathrm{d} x=\int_{\mathbb{R}^{N}} f\left(x, u_{n}\right) u_{n} \mathrm{~d} x+\mathrm{o}(1) .
$$

Since $t_{n} \rightarrow T$, by $\left(f_{1}\right)$ and $\left(f_{3}\right)$

$$
T^{2} \int_{\mathbb{R}^{N}}\left(\left|\nabla u_{n}\right|^{2}+V(x)\left|u_{n}\right|^{2}\right) \mathrm{d} x-\int_{\mathbb{R}^{N}} f\left(x, T u_{n}\right) T u_{n} \mathrm{~d} x=\mathrm{o}(1),
$$

that is

$$
\mathrm{o}(1)=\int_{\mathbb{R}^{N}} \frac{f\left(x, T u_{n}\right)}{T u_{n}} u_{n}^{2}-\frac{f\left(x, u_{n}\right)}{u_{n}} u_{n}^{2} \mathrm{~d} x=\int_{\mathbb{R}^{N}}\left(\frac{f\left(x, T u_{n}\right)}{T u_{n}}-\frac{f\left(x, u_{n}\right)}{u_{n}}\right) u_{n}^{2} \mathrm{~d} x .
$$

For a subsequence $u_{n} \rightarrow u$ in $L_{\text {loc }}^{p}\left(\mathbb{R}^{N}\right) 2 \leqslant p<2^{*}$. Up to translations, we may assume $u \neq 0$. Then by $\left(f_{4}\right)$ and Fatou's lemma

$$
\int_{\mathbb{R}^{N}}\left(\frac{f(x, T u)}{T u}-\frac{f(x, u)}{u}\right) u^{2} \mathrm{~d} x=0,
$$

by $\left(f_{4}\right)$ we have $T=1$.

Next we construct a special minimizing sequence along which $\int_{\mathbb{R}^{N}} F(x, u)$ is weakly continuous. Consider Eq. (1.1) on $B_{R}(0)$,

$$
\begin{cases}-\Delta u+V(x) u=f(x, u), & \text { in } B_{R}(0) \\ u=0, & \text { on } \partial B_{R}(0)\end{cases}
$$

We can similarly define $\mathcal{N}_{R}, c_{R}$. By the result of [8], $c_{R}$ is achieved by a positive solution of (2.1) called $u_{R}$. It is easy to check that $c_{R}>c$ and $c_{R} \rightarrow c$ as $R \rightarrow \infty$. This implies $\left(u_{R}\right)$ as $R \rightarrow \infty$ minimizes $c$. Let $R_{n} \rightarrow \infty, u_{n}:=u_{R_{n}}$. Fix $p \in\left(2,2^{*}\right)$.

\section{Lemma 2.5.}

(i) $\int_{\mathbb{R}^{N}}\left|u_{n}\right|^{p} \rightarrow A>0$.

(ii) There exist $x_{n} \in \mathbb{R}^{N}$ such that $\forall \varepsilon>0, \exists R>0$, $\liminf \int_{B_{R}\left(x_{n}\right)}\left|u_{n}\right|^{p} \geqslant A-\varepsilon$.

Proof. (i) follows from $\gamma\left(u_{n}\right)=0$ and the fact that for any $\varepsilon>0$ there is $C_{\varepsilon}>0,|f(x, u)| \leqslant \varepsilon\left(|u|+|u|^{2^{*}-1}\right)+$ $C_{\varepsilon}|u|^{p-1}$.

For (ii) we apply the concentration compactness principle to $\int_{\mathbb{R}^{N}}\left|u_{n}\right|^{p}$. Then there exist $\alpha \in(0,1],\left(x_{n}\right) \subset \mathbb{R}^{N}$, $\forall \varepsilon>0, \exists R>0, \forall r>R, r^{\prime}>R$, have

$$
\liminf \int_{B_{r}\left(x_{n}\right)}\left|u_{n}\right|^{p} \geqslant \alpha A-\varepsilon, \quad \liminf \int_{B_{r^{\prime}}^{c}\left(x_{n}\right)}\left|u_{n}\right|^{p} \geqslant(1-\alpha) A-\varepsilon .
$$

Next we claim $\alpha=1$. Choose $\varepsilon_{n} \rightarrow 0, r_{n} \rightarrow \infty, r_{n}^{\prime}=4 r_{n}$. Let $\xi$ be a cut-off function such that $\xi(s)=0$, for $s \leqslant 1$ or $s \geqslant 4, \xi(s)=1$, for $2 \leqslant s \leqslant 3$, and $\left|\xi^{\prime}(s)\right| \leqslant 2$. Take $\phi(x)=\xi\left(\left|x-x_{n}\right| / r_{n}\right) u_{n}$. Using equation

$$
\int_{B_{R_{n}}}\left(\nabla u_{n} \nabla \phi+V(x) u_{n} \phi-f\left(x, u_{n}\right) \phi\right) \mathrm{d} x=0,
$$


we have,

$$
\int_{B_{3 r_{n}}\left(x_{n}\right) \backslash B_{2 r_{n}}\left(x_{n}\right)}\left(\left|\nabla u_{n}\right|^{2}+V(x)\left|u_{n}\right|^{2}\right) \mathrm{d} x+\int_{B_{3 r_{n}}\left(x_{n}\right) \backslash B_{2 r_{n}}\left(x_{n}\right)} f\left(x, u_{n}\right) u_{n} \mathrm{~d} x=\mathrm{o}(1) .
$$

Take another cut-off function $\eta$ such that $\eta(s)=1$, for $s \leqslant 2, \eta(s)=0$, for $s \geqslant 3$, and $\left|\eta^{\prime}(s)\right| \leqslant 2$, for $2 \leqslant s \leqslant 3$. Set

$$
w_{n}(x)=\eta\left(\frac{\left|x-x_{n}\right|}{r_{n}}\right) u_{n}, \quad v_{n}(x)=\left(1-\eta\left(\frac{\left|x-x_{n}\right|}{r_{n}}\right)\right) u_{n}(x) .
$$

Using equation as above we have

$$
\Phi\left(u_{n}\right)=\Phi\left(w_{n}\right)+\Phi\left(v_{n}\right)+\mathrm{o}(1),
$$

and

$$
\int_{\mathbb{R}^{n}}\left|w_{n}\right|^{p} \geqslant \alpha A-\varepsilon_{n}, \quad \int_{\mathbb{R}^{n}}\left|v_{n}\right|^{p} \geqslant(1-\alpha) A-\varepsilon_{n} .
$$

Finally using $w_{n}$ to test the equation for $\left(u_{n}\right)$ we get

$$
\gamma\left(w_{n}\right)=\left\langle\Phi^{\prime}\left(u_{n}\right), w_{n}\right\rangle+\mathrm{o}(1)=\mathrm{o}(1) .
$$

Similarly $\gamma\left(v_{n}\right)=\mathrm{o}(1)$, by Lemma $2.4, \exists t_{n} \rightarrow 1, s_{n} \rightarrow 1$, such that $t_{n} w_{n} \in \mathcal{N}, s_{n} v_{n} \in \mathcal{N}$. Then

$$
c+\mathrm{o}(1)=\Phi\left(u_{n}\right)=\Phi\left(w_{n}\right)+\Phi\left(v_{n}\right)+\mathrm{o}(1)=\Phi\left(t_{n} w_{n}\right)+\Phi\left(s_{n} v_{n}\right)+\mathrm{o}(1) \geqslant 2 c+\mathrm{o}(1),
$$

which is a contradiction. Thus $\alpha=1$.

Proof of Theorem 2.1. Let $\left(u_{n}\right) \subset \mathcal{N}$ be the minimizing sequence for $c$ given above. By Lemma $2.2\left(u_{n}\right)$ is bounded in $X$ and weak convergent to $u \neq 0$. By Lemma 2.5, $-\int_{R^{N}} F\left(x, u_{n}\right)$ is weakly continuous. Using the weakly lower semi-continuity we have $\Phi(u) \leqslant c$. If $u \in \mathcal{N}$ we have $\Phi(u)=c$. If $u \notin \mathcal{N}$, by Lemma 2.5, there is $t>0$ such that $t u_{n} \in \mathcal{N}$. Then

$$
c \leqslant \Phi(t u) \leqslant \liminf _{n \rightarrow \infty} \Phi\left(t u_{n}\right) \leqslant \liminf _{n \rightarrow \infty} \Phi\left(u_{n}\right)=c .
$$

Since $\mathcal{N}$ is smooth, the minimizer is a critical point of $\Phi$.

\section{The potential well case}

We consider weak solutions of

$$
\left\{\begin{array}{l}
-\Delta u+V(x) u=f(u) \\
u \in H^{1}\left(\mathbb{R}^{N}\right)
\end{array}\right.
$$

for the case where potential function $V(x)$ has a bounded potential well. Since the nonlinearity is autonomous, the conditions on $f$ needs modified slightly. More precisely, we make the following assumptions.

$\left(V_{2}\right) \quad 0<\inf _{\mathbb{R}^{N}} V(x) \leqslant \lim _{|x| \rightarrow \infty} V(x)=\sup _{\mathbb{R}^{N}} V(x)<\infty$.

( $\left.f_{1}\right) f(t) \in C^{1} . f_{t}$ is a Caratheodory function and there exists $C>0$, s. t.

$$
\left|f_{t}(t)\right| \leqslant C\left(1+|t|^{2^{*}-2}\right), \quad \lim _{|t| \rightarrow \infty} \frac{|f(t)|}{|t|^{2^{*}-1}}=0 .
$$

$\left(f_{2}\right) \quad f(t)=\mathrm{o}(|t|)$, as $|t| \rightarrow 0$.

(f $\left.f_{3}\right) \lim _{|t| \rightarrow \infty} \frac{F(t)}{t^{2}}=\infty$.

$\left(f_{4}\right) \frac{f(t)}{|t|}$ is strictly increasing in $t$. 
Theorem 3.1. Under assumptions $\left(V_{2}\right),\left(f_{1}\right)-\left(f_{4}\right) E q$. (3.1) has a weak solution $u \in X$, such that $\Phi(u)=c>0, c$ is defined as

$$
c=\inf _{\mathcal{N}} \Phi(u)
$$

where $\mathcal{N}=\{u \in X: u \neq 0, \gamma(u)=0\}$.

In this section we denote $V_{\infty}=\lim _{|x| \rightarrow \infty} V(x)$. There is an associated problem

$$
\left\{\begin{array}{l}
-\Delta u+V_{\infty} u=f(u), \\
u \in H^{1}\left(\mathbb{R}^{N}\right) .
\end{array}\right.
$$

We define the energy functional $\Phi_{\infty}$ by replacing $V$ with $V_{\infty}, c_{\infty}=\inf _{\mathcal{N}_{\infty}} \Phi_{\infty}(u)$, here $\mathcal{N}_{\infty}=\{u \in X /\{0\}$ : $\left.\left\langle\Phi_{\infty}^{\prime}(u), u\right\rangle=0\right\}$. Since $V_{\infty}$ is a constant, by Theorem 2.1, $c_{\infty}>0$ is achieved at some $u_{\infty} \in \mathcal{N}_{\infty}$.

Lemma 3.2. $0<c<c_{\infty}$.

Proof. It is easy to see $c>0$. Let $u_{\infty}$ be the minimizer of $c_{\infty}$. Then $\gamma\left(u_{\infty}\right)<0$, and there is $t>0$ such that $t u_{\infty} \in \mathcal{N}$. We have

$$
c \leqslant \Phi\left(t u_{\infty}\right)<\Phi_{\infty}\left(t u_{\infty}\right) \leqslant \Phi_{\infty}\left(u_{\infty}\right)=c_{\infty} .
$$

We note that with minor changes Lemma 2.3 and 2.4 still hold.

Lemma 3.3. Let $\left(u_{n}\right)$ be a minimizing sequence for $c$. Then

(i) There is $\beta>0$ such that $\liminf _{n \rightarrow \infty}\left\|u_{n}\right\| \geqslant \beta$.

(ii) $\left(u_{n}\right)$ is bounded in $X$.

(iii) For a subsequence, $u_{n}$ converges weakly to $u \neq 0$.

Proof. (i) The same as Lemma 2.2.

(ii) If not, define $v_{n}=u_{n} /\left\|u_{n}\right\|$. Passing to a subsequence, we may assume, $v_{n} \rightarrow v$ in $X$. If $v_{n} \rightarrow 0$ in $L^{q}\left(\mathbb{R}^{N}\right)$ for $2 \leqslant q<2^{*}$, we use the Lebesgue Dominated Convergence theorem to get for any $R>0$ fixed, $\lim _{n \rightarrow \infty} \int_{\mathbb{R}^{N}} F\left(R v_{n}\right) \mathrm{d} x=0$. Therefore a contradiction by choosing a large $R>0$ in $\Phi\left(u_{n}\right) \geqslant \Phi\left(R v_{n}\right)=\frac{1}{2} R^{2}-$ $\int_{\mathbb{R}^{N}} F\left(R v_{n}\right) \mathrm{d} x$. Thus by the concentration compactness principle there are $y_{n} \in \mathbb{R}^{N}$ such that $w_{n}(x)=v_{n}\left(y_{n}+x\right) \rightarrow$ $w \neq 0$. Then the proof follows from the arguments in Lemma 2.2(ii). Thus $\left(u_{n}\right)$ is bounded.

(iii) We can assume $u_{n} \rightarrow u$ in $X, u_{n} \rightarrow u$ in $L_{\text {loc }}^{p}\left(\mathbb{R}^{N}\right)$. If $u=0$, we have $\int_{\mathbb{R}^{N}}\left(V(x)-V_{\infty}\right)\left|u_{n}\right|^{2} \mathrm{~d} x \rightarrow 0$, as $n \rightarrow \infty$. Thus we have $c+\mathrm{o}(1)=\Phi_{\infty}\left(u_{n}\right)+\mathrm{o}(1)$. Similarly we have $\gamma\left(u_{n}\right)=0, \gamma_{\infty}\left(u_{n}\right)=\mathrm{o}(1)$. By Lemma 2.4 there exist $t_{n} \rightarrow 1$ such that $t_{n} u_{n} \in \mathcal{N}_{\infty}$. Then we have $c+\mathrm{o}(1)=\Phi_{\infty}\left(u_{n}\right)+\mathrm{o}(1)=\Phi_{\infty}\left(t_{n} u_{n}\right)+\mathrm{o}(1) \geqslant c_{\infty}+\mathrm{o}(1)$, a contradiction with Lemma 3.2.

Next consider Eq. (3.1) on $B_{R}(0)$,

$$
\begin{cases}-\Delta u+V(x) u=f(u), & \text { in } B_{R}(0) \\ u=0, & \text { on } \partial B_{R}(0)\end{cases}
$$

we can similarly define $\mathcal{N}_{R}=\mathcal{N} \cap H_{0}^{1}\left(B_{R}\right), c_{R}$. By the result of [8], $c_{R}$ is achieved by a positive solution called $u_{R}$. It is easy to check that $c_{R}>c$ and $c_{R} \rightarrow c$ as $R \rightarrow \infty$.

Lemma 3.4. Let $u_{R} \in \mathcal{N}_{R}$ be a minimizer of $c_{R}$. Assume for a subsequence $R_{n} \rightarrow \infty, \int_{B_{R_{n}}}\left|u_{n}\right|^{p} \rightarrow A \in(0, \infty)$, where $u_{n}=u_{R_{n}}$. Then there exists $\left(y_{n}\right) \subset \mathbb{R}^{N}$ s.t. for any $\varepsilon>0$, exists $r_{\varepsilon}>0$, for all $r \geqslant r_{\varepsilon}$,

$$
\liminf _{n \rightarrow \infty} \int_{B_{r}\left(y_{n}\right)}\left|u_{n}\right|^{p} \geqslant A-\varepsilon .
$$


Proof. Note that $u_{R}$ satisfies (3.3) for all $\varphi \in H_{0}^{1}\left(B_{R}\right)$,

$$
\int_{B_{R_{n}}}\left(\nabla u_{R} \nabla \varphi+V(x) u_{R} \varphi\right) \mathrm{d} x-\int_{\mathbb{R}^{N}} f\left(u_{R}\right) \varphi \mathrm{d} x=0 .
$$

Since $\left(u_{n}\right)$ is bounded in $H^{1}\left(\mathbb{R}^{N}\right)$, by using the concentration compactness principle, exists $\alpha \in(0,1]$ and $\left(y_{n}\right) \subset \mathbb{R}^{N}$ s.t. for any $\varepsilon>0$, there exists $r_{\varepsilon}>0$, for all $r^{\prime} \geqslant r \geqslant r_{\varepsilon}$,

$$
\begin{aligned}
& \liminf _{n \rightarrow \infty} \int_{B_{r}\left(y_{n}\right)}\left|u_{n}\right|^{p} \geqslant \alpha A-\varepsilon, \\
& \liminf _{n \rightarrow \infty} \int_{\mathbb{R}^{N} \backslash B_{r^{\prime}}\left(y_{n}\right)}\left|u_{n}\right|^{p} \geqslant(1-\alpha) A-\varepsilon .
\end{aligned}
$$

Now suppose $\alpha<1$, then following exactly the same construction as in Lemma 2.5 we have two sequences $w_{n}$ and $v_{n}$ satisfying

$$
\liminf _{n \rightarrow \infty} \int_{\mathbb{R}^{N}}\left|w_{n}\right|^{p} \geqslant \alpha A, \quad \liminf _{n \rightarrow \infty} \int_{\mathbb{R}^{N}}\left|v_{n}\right|^{p} \geqslant(1-\alpha) A, \quad \Phi\left(u_{n}\right)=\Phi\left(w_{n}\right)+\Phi\left(v_{n}\right)+\mathrm{o}(1) .
$$

Moreover, if we take $\varphi=w_{n}$, by (3.3)

$$
\gamma\left(w_{n}\right)=\left\langle\Phi^{\prime}\left(u_{n}\right), w_{n}\right\rangle+\mathrm{o}(1)=\mathrm{o}(1) .
$$

Similarly, $\gamma\left(v_{n}\right)=\mathrm{o}(1)$. By Lemma 2.4, there exist $t_{n} \rightarrow 1$, $s_{n} \rightarrow 1$, s.t.

$$
\widetilde{w}_{n}=t_{n} w_{n} \in \mathcal{N}, \quad \tilde{v}_{n}=s_{n} v_{n} \in \mathcal{N} .
$$

If $\left(y_{n}\right)$ is bounded, then $\liminf _{n \rightarrow \infty} \Phi\left(\widetilde{w}_{n}\right) \geqslant c$ and $\liminf _{n \rightarrow \infty} \Phi\left(\tilde{v}_{n}\right) \geqslant c_{\infty}$. If $\left(y_{n}\right)$ is unbounded, then

$$
\liminf _{n \rightarrow \infty} \Phi\left(\widetilde{w}_{n}\right) \geqslant c \infty \text { and } \liminf _{n \rightarrow \infty} \Phi\left(\tilde{v}_{n}\right) \geqslant c .
$$

Altogether, we have

$$
\Phi\left(u_{n}\right)=\Phi\left(w_{n}\right)+\Phi\left(v_{n}\right)+\mathrm{o}(1)=\Phi\left(t_{n} w_{n}\right)+\Phi\left(s_{n} v_{n}\right)+\mathrm{o}(1),
$$

and

$$
\liminf \Phi\left(u_{n}\right) \geqslant \liminf \Phi\left(t_{n} w_{n}\right)+\liminf \Phi\left(s_{n} v_{n}\right) \geqslant c+c_{\infty}
$$

A contradiction, so we have $\alpha=1$.

Proof of Theorem 3.1. Let $\left(u_{n}\right) \subset \mathcal{N}$ be the minimizing sequence for $c$ given in Lemma 3.4. Let $A=$ $\lim _{n \rightarrow \infty} \int_{\mathbb{R}^{N}}\left|u_{n}\right|^{p} \mathrm{~d} x$. By Lemma 3.3, $\left(u_{n}\right)$ is bounded in $X$ and weakly converges to $u \neq 0$. By Lemma 3.4, there exists $\left(y_{n}\right) \subset \mathbb{R}^{N}$ s.t. $\forall \varepsilon>0, \exists r>0$,

$$
\liminf _{n \rightarrow \infty} \int_{B_{r}\left(y_{n}\right)}\left|u_{n}\right|^{p} \geqslant A-\varepsilon .
$$

Then $\left(y_{n}\right)$ must be bounded. Otherwise, $\gamma_{\infty}\left(u_{n}\right)=\gamma\left(u_{n}\right)+\mathrm{o}(1)$. We find $t_{n} \rightarrow 1$, s.t. $\gamma\left(t_{n} u_{n}\right)=0$. Then we have

$$
c_{\infty} \leqslant \liminf \Phi_{\infty}\left(t_{n} u_{n}\right)=\liminf \Phi_{\infty}\left(u_{n}\right)=\liminf \Phi\left(u_{n}\right)=c
$$

a contraction with $c<c_{\infty}$. Now, when $\left(y_{n}\right)$ is bounded, we have $u_{n} \rightarrow u$ in $L^{p}\left(\mathbb{R}^{N}\right)$. This gives that along this sequence $\Phi\left(u_{n}\right)$ is weakly lower semi-continuous, we have

$$
c=\inf _{\mathcal{N}} \Phi(u) \leqslant \Phi(u) \leqslant \liminf \Phi\left(u_{n}\right)=c .
$$

Remark 3.5. Though we assume in this section $f$ depends only on $t$, looking at the proofs we see the arguments can be used with little changes to deal with the following case: $f=b(x) f(t)$ with $b$ satisfying $b \in C^{1}\left(\mathbb{R}^{N}, \mathbb{R}\right)$, $b_{1} \leqslant b(x) \leqslant b_{2}$ for some $b_{1}, b_{2}>0$, and $b(x) \geqslant \inf _{\mathbb{R}^{N}} b(x)=\lim _{|x| \rightarrow \infty} b(x)$. The precisely statement is the same. 


\section{References}

[1] A. Ambrosetti, A. Malchiodi, Perturbation Methods and Semilinear Elliptic Problems on $\mathbb{R}^{n}$, Progr. Math., Birkhäuser, in press.

[2] A. Ambrosetti, P.H. Rabinowitz, Dual variational methods in critical point theory and applications, J. Funct. Anal. 14 (1973) $349-381$.

[3] H. Berestycki, P.L. Lions, Nonlinear scalar field equations. I. Existence of a ground state, Arch. Rational Mech. Anal. 82 (1983) $313-345$.

[4] W.-Y. Ding, W.-M. Ni, On the existence of positive entire solutions of a semilinear elliptic equation, Arch. Rational Mech. Anal. 91 (1986) 283-308

[5] L. Jeanjean, K. Tanaka, A positive solution for a nonlinear Schrödinger equation on $\mathbb{R}^{N}$, Indiana Univ. Math. J. 54 (2005) $443-464$.

[6] P.L. Lions, The concentration-compactness principle in the calculus of variations. The locally compact case. I \& II, Ann. Inst. H. Poincaré Anal. Non Linéaire 1 (1984) 109-145, 223-283.

[7] J. Liu, Y. Wang, Z.-Q. Wang, Solutions for quasilinear Schrödinger equations via the Nehari method, Comm. Partial Differential Equations 29 (2004) 879-901

[8] Z. Liu, Z.-Q. Wang, On the Ambrosetti-Rabinowitz superlinear condition, Adv. Nonlinear Stud. 4 (2004) 561-572.

[9] P.H. Rabinowitz, On a class of nonlinear Schrödinger equations, Z. Angew. Math. Phys. 43 (1992) 270-291.

[10] W.A. Strauss, Existence of solitary waves in higher dimensions, Comm. Math. Phys. 55 (1977) 149-162.

[11] M. Struwe, Variational Methods. Applications to Nonlinear Partial Differential Equations and Hamiltonian Systems, Springer-Verlag, Berlin, 2000.

[12] M. Willem, Minimax Theorems, Birkhäuser, Boston, 1996. 